(Aus dem neuro-biologischen Laboratorium [E. Flatau] der Warschauer Gesellschaft der Wissenschaften.)

\title{
Histologische Veränderungen im Nervensystem bei experimenteller Thyreotoxikose.
}

\author{
Von \\ Teofil Simchowicz, \\ Assistent am neuro-biologischen Laboratorium der Warschauer Gesellschaft der Wissenschaften.
}

Mit 4 Textfiguren und 6 Tafeln.

(Eingegangen am 25. Juli 1914.)

Das Ziel der vorliegenden Untersuchungen war, festzustellen, ob bei Säugetieren durch Einverleibung von Blutdrüsenextrakten greifbare histologische Veränderungen im Nervensystem erzeugt werden können.

Die zahlreichen Untersuchungen der letzten Jahre haben gezeigt, $\mathrm{da} B$ das Nervensystem in hervorragender Weise von den Produkten der inneren Sekretion abhängig ist, und daß zwischen Erkrankungen des Nervensystems und Störungen der endokrinen Drüsen enge Beziehungen bestehen können.

Während wir auf diesem Gebiete eine ganze Reihe von klinischen und klinisch-experimentellen Arbeiten besitzen, gibt es bis jetzt fast gar keine histologischen Untersuchungen über Veränderungen im Nervensystem, welche durch Produkte der inneren Sekretion erzeugt sind. In der ganzen diesbezüglichen Literatur konnte ich nur zwei Arbeiten auffinden, welche dieses Thema behandeln. Es sind die Untersuchungen von Erb jun. und von Shima, die sich mit den Veränderungen des Zentralnervensystems bei Kaninchen nach Adrenalinjektionen beschäftigt haben. Aber gerade das Adrenalin eignet sich am wenigsten zur Feststellung der toxischen Veränderungen im Nervensystem, und zwar deshalb, weil es schwere Veränderungen im Gefäßsystem und Hämorrhagien erzeugt. Die Veränderungen der Ganglienzellen, der Glia, ebenso wie die Plasmazellen-Infiltrate, welche von beiden Autoren in der Pia und in einzelnen Gefäßscheiden des Gehirns festgestellt worden sind, sind wohl im wesentlichen als Folgeerscheinungen der Gehirnblutungen zu deuten, um so mehr, als die Veränderungen am stärksten in der Umgebung der hämorrhagischen Herde ausgesprochen waren, während die Nervenzellen in den von der Blutung weiter entfernten Rindenpartien ein fast normales Aussehen zeigten. 
Außer diesen zwei Arbeiten hat noch die Arbeit von Birch - Hirsch feld und Nobuo-Inou ye (Experimentelle Untersuchungen über die Pathogenese der Thyreoidin-Amblyopie) ein gewisses Interesse für uns. Diese Autoren konnten bei Hunden durch fortgesetzte Zufuhr von Thyreoidin eine Sehnervenatrophie mit anatomisch nachweisbaren Degenerationserscheinungen der Netzhaut und der Sehnerven erzeugen. Mit diesen drei Studien ist die ganze uns direkt interessierende Literatur erschöpft.

Unsere Experimente wurden von der Thyreoidea, als der in der Neurologie eine besonders wichtige Rolle spielenden Blutdrüse, begonnen.

Die Versuche wurden an 42 Kaninchen und 9 Hunden ausgeführt. Das Thyreoidin wurde in Pulverform angewandt, da es leichter dosierbar ist (Thyreoidinum siccum Gehe*). 25 Kaninchen wurde das Thyreoidin als wässerige Emulsion vermittels eines Katheters in täglichen Dosen von 0,05 bis $3 \mathrm{~g}(0,015$ bis 1,6 pro Kilo) verfüttert.

3 Kaninchen wurde die wässerige Thyreoidinemulsion subcutan in Dosen von 0,15 bis 0,25 pro Kilo injiziert. 4 Hunden wurde das Thyreoidin in Milch oder mit weicher Wurst vermischt in Dosen von $1-6 \mathrm{~g}$ pro Kilo verabreicht; außerdem wurde die Thyreoidinemulsion 5 Hunden in Dosen von 0,2-1 g pro Kilo subcutan injiziert; 1 Hund und $1 \mathrm{Ka}$ ninchen wurden mit von Basedowkranken entnommenen Schilddrüsen verfüttert**). Um dem eventuellen Vorwurf zuvorzukommen, daß es sich bei diesen Experimenten um Vergiftungen mit heterogenem Tiereiweiß oder mit dem im Thyreoidin enthaltenem Jod handeln könnte, wurden an 13 Kaninchen Kontrollversuche ausgeführt. 3 Kaninchen wurde Hühnereiweiß (1-2 Eier pro dosi) verfüttert, 2 Kaninchen in derselben Dosis subcutan injiziert, 1 Kaninchen wurde mit Liebigs Fleischextrakt, 2 mit Rindfleisch (bis $15 \mathrm{~g}$ täglich pro Kilo), 2 mit Schweinsleber (bis $15 \mathrm{~g}$ pro Kilo täglich) längere Zeit verfüttert.

Außerdem wurde 3 Kaninchen Jodkali in 10, ev. 20proz. Lösung subcutan in Dosen von 0,05-1,0 Jodkali pro Kilo täglich injiziert.

Näheres über die Versuchsanordnungen wird in beigefügten Tabellen angegeben. Wir haben deswegen die Schilddrüsensubstanz den Tieren hauptsächlich verfüttert, weil auf diese Weise eine Reihe von Fehlerquellen vermieden wird. Sobald sich die Toxizität eines Organextraktes nur bei wiederholter subcutaner Einverleibung manifestiert, kann es

*) Bei 3 Kaninchen und 2 Hunden wurde ein anderes Thyreoidinpräparat, nämlich die Tabletten von Burrough Wellcome angewandt; die klinischen Erscheinungen und die histologischen Veränderungen waren genau dieselben wie bei der Vergiftung mit Thyreoidinum siccum Gehe.

**) Bei beiden Tieren wurden außer einer unerheblichen Gewichtsabnahme keine toxischen Erscheinungen wahrgenommen, vielleicht weil wir über zu wenig Schilddrüsenmaterial von Basedowkranken verfügten. 
sich um eine anaphylaktische Reaktion handeln; andererseits, falls toxische Erscheinungen bei intravenöser Injektion der Organextrakte hervortreten, kann das Versuchsergebnis durch die in den Extrakten enthaltenen gerinnungsfördernden Stoffe, sog. Thrombokinasen, erheblich beeinflußt werden. Die intravasculären Gerinnungen können ausgesprochene Funktionsstörungen der Zirkulation und Respiration hervorrufen, ja die Tiere können sogar ganz akut zugrunde gehen (Biedl).

Endlich muß bei subcutaner oder intravenöser Einverleibung auch darauf geachtet werden, ob die injizierten Extrakte vollständig steril sind.

Viel mehr Vertrauen verdienen deshalb die Experimente, in denen Schilddrüsensubstanz den Tieren verfüttert wird, und wir können bei dieser Versuchsweise die toxischen Erscheinungen mit größerem Recht auf spezifische Wirkung des Thyreoidins zurückführen.

Die klinischen Symptome, welche wir bei 25 mit Thyreoidin verfütterten und bei 3 mit Thyreoidinemulsion subcutan injizierten Kaninchen beobachten konnten, waren ziemlich einheitlich. Die konstantesten Symptome waren Abmagerung, Haarausfall und Pulsbeschleunigung. Exophthalmus konnte nur in einem Falle beobachtet werden (Kaninchen VIII). Bei eînzelnen Kaninchen traten Diarrhöen auf. Bei 4 Kaninchen wurde der Urin auf Zucker untersucht; Glykosurie konnte in keinem dieser Fälle festgestellt werden, auch Albuminurie fehlte in allen untersuchten Fällen. Das hervorstechendste klinische Symptom war die Abmagerung, die in keinem einzigen Falle fehlte. Gewöhnlich konnte sie schon am nächsten Tage nach der ersten Fütterung festgestellt werden, nur ausnahmsweise dauerte es einige Tage, bis eine deutliche Gewichtsabnahme auftrat. In zwei Fällen, wo nach längerer Fütterung keine erhebliche Gewichtsabnahme, ja sogar in einem Falle eine Gewichtszunahme festgestellt werden konnte, erwies sich, daß die Kaninchen gravid waren. In beiden diesen Fällen lebten die Kleinen nur ganz kurz, und die Kaninchen selbst sind ebenfalls bei weiterer Thyreoidinfütterung unter rapider Abmagerung zugrunde gegangen.

Zwischen der Thyreoidindose und der Gewichtsabnahme bestanden enge Beziehungen, obwohl die Abmagerung keineswegs proportionell der Thyreoidindose vor sich ging. Kaninchen, die 0,025 Thyreoidin pro Kilo Gewicht erhalten haben, verloren täglich ebenfalls pro Kilo ungefähr $10 \mathrm{~g}$, bei 0,05 pro Kilo $20 \mathrm{~g}$, bei 1,0 pro Kilo 45 pro Kilo täglich, bei 1,6 pro Kilo ungefähr $60 \mathrm{~g}$ pro Kilo täglich. Wir sehen also, daß, während die Thyreoidindose pro Kilo um das 30 fache wächst, die Gewichtsabnahme ebenfalls pro Kilo nur um das Sechsfache gestiegen ist.

Es genügte, die Fütterung für einen Tag einzustellen, um am darauffolgenden Tage eine Gewichtzunahme von $20-100 \mathrm{~g}$ zu erhalten. 
Ein zweites konstantes Symptom ist die Tachykardie. Bei größeren Dosen konnte die Pulsbeschleunigung schon nach 2-3 Tagen, bei kleineren Dosen nach 8-10 Tagen deutlich festgestellt werden. Die letzten Tage vor dem Tode war der Puls bei den Kaninchen kaum mehr zählbar.

Das dritte Symptom - der Haarausfall - fehlte in keinem einzigen Falle, stellte sich gewöhnlich nach 5-6 Tagen (bei kleinen Dosen noch später) ein und blieb bis zum Tode bestehen. Die Haare wurden ungemein lose, so daß beim leichtesten Zupfversuch zwischen den Fingern ein dicker Haarbüschel blieb.

Von den 25 mit Thyreoidin gefütterten Kaninchen wurden 13 in verschiedenen Stadien der Thyreoidinvergiftung getötet, die übrigen 12 gingen alle unter mehr oder weniger rapidem Absinken ihres Körpergewichts zugrunde. Die Lebensdauer war desto länger, je größer die Kaninchen, und je kleiner die Thyreoidindose war. Bei großen Dosen ( $1 \mathrm{~g}$ und mehr täglich pro Kilo) gingen die Tiere schon nach 7-12 Tagen zugrunde, wobei sie $\mathbf{1 6 - 4 5 \%}$ ihres Körpergewichts verloren haben.

Bei kleinen Dosen $(0,015-0,05$ pro Kilo täglich) lebten die Kaninchen 22-36 Tage, wobei sie 35-51\% ihres Gewichtes verloren haben. Auch bei größeren Dosen konnten die Kaninchen länger am Leben erhalten werden, falls die Fütterung nicht jeden Tag stattfand. So lebte das Kaninchen XXIV 18 Tage, während deren es 10 mal zu $2 \mathrm{~g}$ Thyreoidin erhielt.

Die drei mit Thyreoidinemulsion injizierten Kaninchen*) gingen zwischen dem 10.-26. Tage zugrunde, nachdem sie $22-32 \%$ ihres Körpergewichts verloren haben.

Alle 28 Kaninchen waren die ersten 2-4 Tage lebhaft und hatten guten Appetit, erst später nahm der Appetit ab, die Kaninchen wurden unbeweglich und apathisch und blieben so bis zum Tode.

Bei einem Kaninchen konnten während der Agonie tonische Zukkungen im ganzen Körper beobachtet werden.

Hunde reagierten auf das Thyreoidin in einer ganz anderen Weise, als Kaninchen. Tägliche Dosen von 0,3-1,0 pro Kilo Gewicht erzeugten bei ihnen keine wahrnehmbaren klinischen Erscheinungen. Die Hunde, welche eine Woche täglich zu 3-6 g Thyreoidin verzehrten, befanden sich völlig gut und zeigten keine Gewichtsabnahme, erst nachdem wir bedeutend größere Dosen einzuführen begannen, von $12 \mathrm{~g}$ täglich an $\left(1 \frac{1}{2}\right.$ g täglich pro Kilo Gewicht) gelang es uns, eine unbedeutende Gewichtsabnahme nebst Aufregung hervorzurufen. Ausgesprochene Vergiftungssymptome setzten bei Hunden erst nach Anwendung von

*) Diese 3 Kaninchen wurden mir vom Kollegen Stefan Sterling, der sie für andere Zwecke brauchte, zur Verfügung gestellt, wofür ich ihm an dieser Stelle nochmals meinen besten Dank ausdrücke. 
ungeheuren Dosen ein (30-100 g täglich; 4-15 g pro Kilo Körpergewicht des Tieres), wobei diese Erscheinungen einen anderen Charakter trugen, als bei Kaninchen.

Im ersten Stadium pflegte der Hund aufgeregt zu sein, konnte keinen Augenblick ruhig bleiben; daraufhin wurde er apathisch, hatte fortwährend Erbrechen, wies jegliches Futter ab, mit Ausnahme von Wasser und Milch, die er aber ebenfalls sofort erbrach. Die Herztätigkeit war ausdrücklich beschleunigt. Der Puls überschritt 200 Schläge pro Minute und wurde zuletzt unzählbar. Unbedeutender Haarausfall konnte ebenfalls festgestellt werden. Tod trat ein nach mehrtägigem Hungern unter Erscheinungen von allgemeiner Kachexie und bei Gewichtsabnahme von $30-35 \%$.

Bei Erwägung der Tatsache, daß Kaninchen gegen die geringste, in den Darmkanal eingeführte Thyreoidindose ungemein empfindlich sind, wogegen Hunde auf bedeutend größere Dosen nicht reagieren, versuchten wir uns dieselbe in der Weise zu erklären, daß die Kaninchen, als Grasfresser, im Darmkanal keine Substanzen besitzen, welche sie vor dem im Thyreoidin enthaltenen tierischen Eiweiß schützen könnten, wogegen der Hund, der ein Fleischfresser ist, in seinem Verdauungskanal Schutzsubstanzen besitzt, welche das Thyreoidintoxin in für den Organismus unschädliche Bestandteile zerlegen.

Um diese Voraussetzung bestätigen zu können, versuchten wir, Kaninchen und Hunden subcutan Thyreoidin einzuspritzen (in Form von Emulsion in physiologischer Lösung). Kaninchen reagierten in gleicher Weise, wie bei Einführung des Thyreoidins in den Magen; nach einigen Injektionen begannen sie bereits Gewichtsabnahme zu zeigen, und nach mehreren gingen sie zugrunde unter den gleichen Erscheinungen, die bei Kaninchen, denen Thyreoidin in den Magen eingeführt wurde, beobachtet worden sind.

Hunde ertrugen ohne wahrnehmbare Reaktion wiederholte subcutane Injektionen von $1 \mathrm{~g}$ Thyreoidin. Angesichts dessen spritzte ich 2 Hunden je $4 \mathrm{~g}$ Thyreoidin ein. Bei einem derselben bildete sich an der Injektionsstelle ein Absceß, der Hund begann zu fiebern und ging nach 10 Tagen zugrunde. Beim zweiten Hunde traten Erscheinungen auf, auf welche wir näher eingehen möchten.

Der nach der Injektion entstandene Tumor wurde allmählich kleiner, zeigte keine Schmerzhaftigkeit; Temperatur blieb stets normal.

Nach 10 Tagen begann der Hund abzumagern, obgleich er immer gute Eßlust hatte und gehörig fraß.

15. IX. 1914 (vor der Injektion) Gewicht $3800 \mathrm{~g}$

18. IX. $1914,, \quad, \quad, \quad, \quad 3800 \mathrm{~g}$

22. IX. $1914 \quad, \quad, \quad, \quad, \quad 3800 \mathrm{~g}$

25. IX. $1914 \quad, \quad, \quad,, \quad, \quad 3650 \mathrm{~g}$ 
2. X. 1914 (vor der Injektion) Gewicht $3420 \mathrm{~g}$

18. X. $1914 \quad, \quad, \quad, \quad, \quad 3200 \mathrm{~g}$

20. X. $1914,, \quad, \quad, \quad, \quad 3100 \mathrm{~g}$

24. X. $1914, ", \quad, \quad, \quad 3050 \mathrm{~g}$

29. X. $1914 \quad, \quad " \quad, \quad, \quad 3000 \mathrm{~g}$

13. XI. $1914, ", ", \quad, \quad 2900 \mathrm{~g}$

Am 13. November, also in 8 Wochen nach subcutaner Einspritzung von $4 \mathrm{~g}$ Thyreoidin, konnten außer der allgemeinen Abmagerung (Textfig. 1), ausdrückliche trophische Veränderungen an den Ohren (Tafel XX) und am Schweife (Textfig. 1, 2) wahrgenommen worden. Der vorher normal behaarte distale Schweifteil verlor fast sämtliche

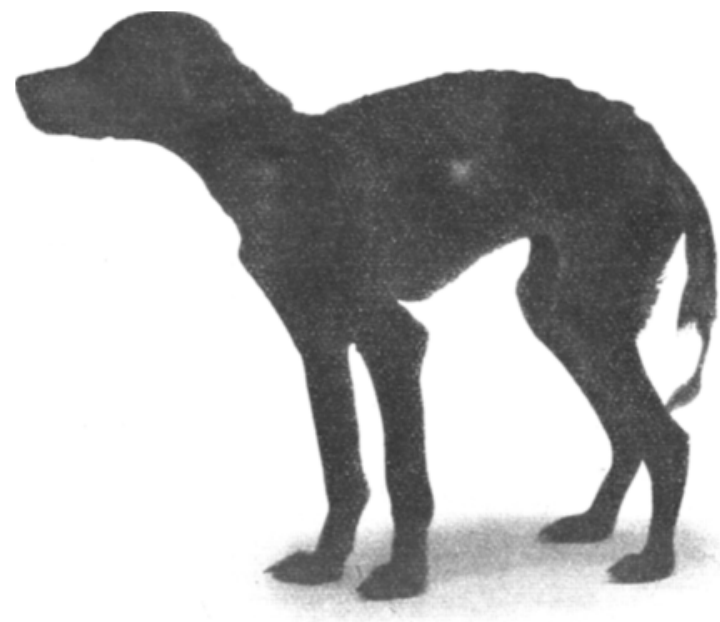

Fig. 1. Hund VII. 21/2 Monate nach subcutaner Injektion von $4 \mathrm{~g}$ Thyreoidin. Allgemeine Abmagerung und schwere trophische Veränderungen am Schweif.

Haare; nur ganz am Ende blieb ein kleiner Haarbüschel zurück (Textfig. 1, 2). Der enthaarte Teil des Schwanzes war völlig dürr und dunkel gefärbt. Der die Grenze zwischen dem gesunden und dem kranken Schwanzteile bildende Wirbel war aufgedunsen und rosig gefärbt. Außer dem Schweifbrande haben wir am oberen Pol beider Ohren zwei ganz symmetrische nekrotische Wunden bemerkt, von ovalem Umriß, $9 \mathrm{~mm}$ Länge, $3 \mathrm{~mm}$ Breite und 1,5 mm Tiefe. Die Schmerzempfindung und der Temperatursinn am Schweif und an Ohren schienen etwas herabgesetzt zu sein (der Hund reagierte subnormal gegen Stechen der betreffenden Stellen mit einer Stecknadel und deren Begießen mit Äther).

Am 17. November fiel der brandige Schweifteil ab; an den Ohrenwunden bildeten sich Krusten, die am nächsten Tage abgefallen sind. 
Das Gewicht nahm ununterbrochen ab, obwohl der Hund sehr viel fraß.

19. XI. Gewicht 2700.

29. XI. Gewicht 2720. Fährt fort, tüchtig zu fressen. Hinkt beim Gehen, besonders schwer kommt dem Tiere das Auf- und Absteigen der Treppe. Die Muskelreaktion der vorderen und hinteren Extremitäten auf faradischen Strom ist vollständig normal. Der Harn enthält we ter Zucker, noch Eiweiß.

Der Hund starb am 10. XII., also 86 Tage nach subcutaner Injektion von $4 \mathrm{~g}$ Thyreoidin. Postmortales Gewicht 2700; somit hat der Hund $29 \%$ seines ursprünglichen Gewichts verloren.

Die Sektion ermittelte keine wahrnehmbaren Veränderungen weder in den inneren Organen, noch im Nervensystem.

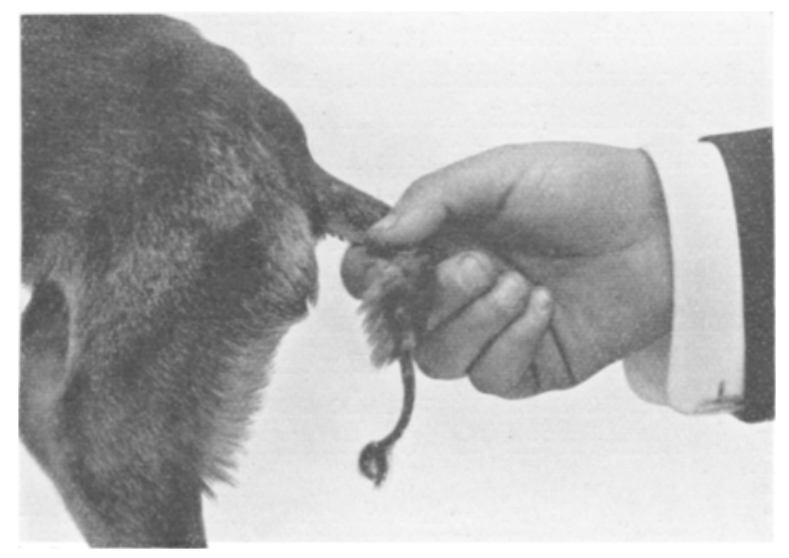

Fig. 2. Hund VII. Trophische Veränderungen am Schweif. Der Wirbel an der Grenze der gesunden und kranken Hälfte ist stark aufgetrieben.

Bei der mikroskopischen Untersuchung des Zentralnervensystems konnten ebenfalls keine Veränderungen nachgewiesen werden.

Um die Gewißheit zu erlangen, ob wir es hier nicht etwa mit einer zufälligen Komplikation zu tun haben, sandten wir den Hund, noch bevor sein Schwanz abgefallen war, in eine Tierklinik. Der Besitzer derselben, Herr Koziolkiewicz, äußerte die Meinung, daß die Veränderungen an Schweif und Ohren von trophischem Charakter sind, und daß er eine derartige Erkrankung weder bei Hunden, noch überhaupt bei Tieren jemals beobachtete und sogar von derselben nie zu hören bekam. Ganz derselben Meinung war auch ein anderer Tierarzt, Herr Dr. Labedz.

Demzufolge glaube ich, daß wir gewissermaßen berechtigt sind, die beim Hunde beobachteten trophischen Veränderungen der Thyreoidin- 
vergiftung zuzuschreiben. Noch stärker befestigte uns in dieser Mainung die Tatsache, daß bald darauf ähnliche, obwohl schwächer ausgesprochene Erscheinungen auch bei einem zweiten Hunde aufzutreten begannen. Bei diesem stellten sich 3 Monate nach Injektion von $3 \mathrm{~g}$ Thyreoidin Haarausfall am Schweifende ein, und an der Peripherie der beiden Ohren bildeten sich zwei symmetrische, oberflächliche Wunden.

Obiger, obwohl weniger demonstrativer Fall befestigte uns in der Meinung, daß die an die Raynaudsche Krankheit erinnernden trophischen Veränderungen durch Thyreoidininjektionen erzeugt wurden, welche anstatt der üblichen, nach Thyreoidinjektionen bei Kaninchen beobachteten Erscheinungen bei Hunden Störungen hervorgerufen haben, die in 2-3 Monaten trophische, an die Rayna udsche Krankheit erinnernde Veränderungen herbeiführten. Wir ließen ebenfalls diesen Umstand nicht außer acht, daß vielleicht das Verbleiben im Keller (derselbe war aber ziemlich hell, geheizt und ventiliert) zur Entstehung der trophischen Veränderungen beigetragen haben mag; gegen diese Voraussetzung spricht jedoch die Tatsache, daß im Laufe der letzten 2 Jahre in demselben Keller mehr als 100 Hunde gehalten wurden, an denen verschiedene Versuche angestellt wurden, und von denen kein einziger dergleichen Veränderungen zeigte.

Weitere bereits eingeleitete Untersuchungen werden vielleicht zur Aufklärung dieser Frage beitragen können.

An diesen Experimenten ist von besonderem Interesse, daß:

1. die Veränderungen an den Ohren eine völlige Symmetrie zeigen (s. Taf. XX),

2. die Untersuchungen des Zentralnervensystems keine Veränderungen ermittelt haben,

3. daß die trophischen Veränderungen nicht in den ersten Tagen nach der Injektion, sondern erst 2-3 Monate später auftraten.

Bezeichnen wir die bei Kaninchen beobachteten Veränderungen als thyreo-toxische, so können die bei Hunden erzeugten trophischen Veränderungen als meta-thyreo-toxische betrachtet werden, da das Thyreoidintoxin offenbar hier nicht sofort auf das Zentralnervensystem wie bei Kaninohen einwirkt, sondern es führt unbekannte Störungen im Organismus herbei, welche sodann sekundär symmetrische trophische Veränderungen in den am schwächsten ernährten, distalsten Teilen des tierischen Organismus, und zwar an Ohren und Schweif erzeugen.

Die histologische Untersuchung von Hirn und Rückenmark in dem von uns erwähnten Fall mit trophischen Veränderungen beim Hunde hat keine Veränderungen ermittelt; andererseits konnten wir bei Hunden, welche es uns durch kolossale Thyreoidindosen zu vergiften gelang, außer einer unbedeutenden Chromatolyse der Nervenzellen nebst 
geringer Gliaproliferation, sowohl im Hirn, als auch im Rückenmark, keine Veränderungen des Nervensystems feststellen.

Da wir in der vorliegenden Arbeit die Untersuchung der spezifischen, durch das Thyreoidin im Nervensystem erzeugten Veränderungen beabsichtigen, so können die bei experimentellen Hunden, welche auch klinisch ganz lifferent auf Thyreoidin reagierten, beobachteten unbedeutenden Veränderungen hier nicht berücksichtigt werden, und beschränken wir uns an dieser Stelle auf eine eingehendere Beschreibung der histologischen Veränderungen im Nervensystem von Kaninchen, welche gegen das Thyreoidintoxin ungemein empfindlich sind.

Makroskopisch konnten im Nervensystem der mit Thyreoidin vergifteten Kaninchen keine Veränderungen festgestellt werden. Erst die mikroskopische Untersuchung hat sehr bedeutende Alterationen des Nervensystems ermittelt.

In meiner vorläufigen, an einer Sitzung der Warschauer Gesellschaft der Wissenschaften vorgetragenen Mitteilung wurde im allgemeinen über die Art der Veränderungen des Nervensystems bei experimenteller Thyreotoxikose berichtet; ich möchte nun hier auf dieselben etwas genauer eingehen.

Histologisch wurde das gesamte Zentralnervensystem der Kaninchen nebst den sympathischen und Spinalganglien untersucht. Das Material wurde in $95 \%$ Alkohol, 10\% Formol, in der Müller schen Flüssigkeit, sowie in der Weigertschen Gliabeize fixiert. Bei der Färbung wurde nach folgenden Methoden verfahren:

1. Thioninfärbung der in Alkohol fixierten Celloidinschnitte von 10-15 m Dicke (Modifikation der Nisslschen Methode).

2. Färbung der in Formalin fixierten Gefrierschnitte nach Bielschowsky.

3. Färbung derselben Schnitte mit Scharlachrot nach Her xhei mer.

4. Färbung derselben Schnitte nach Spielmeyer (Markscheidenfärbung).

5. Färbung der in der Müllerschen Lösung fixierten Celloidinschnitte nach der Weigertschen Markscheidenmethode.

6. Färbung der in Gliabeize oder in 10 proz. Formol fixierten Gefrierschnitte nach Alzheimer (Alzheimersche Methode V mit Hilfe der Mannschen Lösung).

7. Färbung der in der Orthschen Lösung fixicrten Schnitte nach der Methode von Mallory.

Vom Hirn und vom verlängerten Mark wurden Frontalschnitte, vom Rückenmark Quer- und Längsschnitte bereitet. An diesen letzteren Schnitten sind die Veränderungen der weißen Substanz besonders deutlich wahrzunehmen.

Die bedeutendsten Störungen sind im Rückenmark, sowie auch in 
den sympathischen und Spinalganglien beobachtet worden; auch im verlängerten Mark und im Kleinhirn sind ebenfalls bedeutende Veränderungen festgestellt worden; dagegen zeigte die Hirnrinde weniger intensive, obwohl ebenfalls deutliche Alterationen.

Da ich an anderer Stelle die topographische Ausbreitung verschiedener Ganglienzellenerkrankungen, die bei der Thyreotoxikose festgestellt werden konnten, und die genauere Schilderung derselben beabsichtige, so soll hier auf dieselbe nur kurz eingegangen werden.

Die Veränderungen der Ganglienzellen, die wir im Rückenmark der mit Thyreoidin vergifteten Kaninchen beobachten konnten, sind ziemlich mannigfaltig, sie gehören aber zumeist zu den Zellerkrankungen, die Alzheimer als Verflüssigungsvorgänge zusammengefaßt hat.

Im Nissl präparate zeigen sich beinahe alle großen und kleinen Ganglienzellen des Rückenmarks mehr oder weniger lädiert. Viele Ganglienzellen sind abgerundet und leicht geschwollen, die färbbare Niss lsubstanz tritt weniger scharf (Taf. XV, Fig. 3), oder nur an einzelnen Stellen hervor (Taf. XV, Fig. 2), die Fortsätze gehen bei stärkerer Affektion zu Grunde, und die Ganglienzelle erhält die Gestalt einer Kugel (Taf. XV, Fig. 2); mitunter sind die Fortsätze aufgetrieben und intensiv blau gefärbt (Taf. XV, Fig. 1).

Diese Zellveränderung erinnert am meisten an die Nisslsche akute Zellenerkrankung. Der Kern ist bei leichter lädierten Ganglienzellen geschwellt und blaß gefärbt; bei schwerer affizierten nicht mehr sichtbar.

Eine andere, ungemein häufig im Rückenmark der mit Thyreoidin vergifteten Kaninchen beobachtete Ganglienzellerkrankung ist die Nisslsche ,schwere Zellerkrankung", deren verschiedenartige Phasen wir hier beobachten können. Am Anfang erinnern die Zellen an die „,akute Zellerkrankung“, der Zelleib ist leicht geschwellt, die Nisslschollen nicht mehr sichtbar, das Plasma ist feinkörnig, der Kern blaß, das Kernkörperchen intensiv gefärbt (Taf. XV, Fig. 11). In weiteren Stadien sehen wir deutliche Veränderungen am Kern und Kernkörperchen, und zwar Verkleinerung, Schrumpfung und metachromatische Färbung des Kerns und intensivere Färbung des Nucleolus; das in feine Körnchen umgewandelte Protoplasma der Ganglienzelle kann dabei erhalten bleiben (Taf. XV, Fig. 5), oder es beginnt sich um den Kern allmählich aufzulösen (Taf. XV, Fig. 6).

Außer diesen zwei Veränderungen begegneten wir in den schwersten Fällen von Thyreoidinvergiftung einer Ganglienzellveränderung, wie sie meines Wissens in ähnlicher Weise noch nicht beobachtet wurde. Die Nisslschollen sind nicht mehr sichtbar, die ganze Ganglienzelle ist intensiv dunkel gefärbt, zeigt ein ziemlich homogenes Aussehen. Das Protoplasma des Leibes und der weit sichtbaren Fortsätze ist geschrumpft und zackig. Der Kern zeigt tiefe Veränderungen, er wird klein, dunkel, 
hebt sich von der dunklen Umgebung kaum hervor. Die ganze Ganglienzelle erinnert am meisten an einen auf Löschpapier zerfließenden Tintenfleck.

Die schönsten Bilder dieser Zellveränderungen konnten an Längsschnitten des Rückenmarks beobachtet werden (Taf. XV, Fig. 4). Es scheint sich um einen eigenartigen Verflüssigungsprozeß einzelner Ganglienzellen des Rückenmarks zu handeln. Seltener als diese Veränderungen wurden Vakuolisation des Zelleibes (Taf. XV, Fig. 8), körniger Zerfall mit Auftreten der Alzhei merschen „einfach basophilen“ Stoffe in den Protoplasmafortsätzen (Fig. 8) beobachtet. Dabei konnten gelegentlich an den Fortsätzen spindlige, dunkler gefärbte Auftreibungen beobachtet werden (Taf. XV, Fig. 12).

Auch an der Peripherie des Ganglienzellkörpers, in den sog. ,,pericellulären Strukturen" wurden wiederholt Ablagerungen von ,einfach basophilen" Substanzen beobachtet.

Außer diesen Ganglienzellveränderungen konnte vielfach an den kleinen Ganglienzellen des Hinterhorns eine eigentümliche Veränderung beobachtet werden, auf die bei der Schilderung der Ganglienzellen der Molekularschicht der Kleinhirnrinde näher eingegangen werden wird (s. Taf. XVII, Fig. 23-39).

Die Gliazellen der grauen Substanz des Rückenmarks zeigten in den perakuten Fällen die verschiedensten Stadien der amöboiden Umwandlung, wie sie jüngst von Rosental eingehend geschildert wurde. Die zerfallenen Ganglienzellen, wie auch deren Fortsätze sind häufig von zahlreichen Trabantzellen umgeben, die oft amöboide Umwandlung aufweisen (Taf. XV, Fig. 1). Auch im Innern des zerfallenden Zellkörpers können Gliazellen beobachtet werden, so daß auf diese Weise neurophagische Bilder entstehen (Taf. XV, Fig. 1).

Im Bielschowsky-Präparat konnten in den Vorderhornzellen des Rückenmarks mehrfach deutliche Vakuolen festgestellt werden. Um die Vakuolen herum und in den Fortsätzen waren die Neurofibrillen leidlich gut erhalten (Taf. XVI, Fig. 18-21).

Wir gehen nun zu den Veränderungen der weißen Substanz des Rückenmarks über.

An den Achsenzylindern ließen sich keine deutlichen Ausfälle, dagegen aber ausgesprochene morphologische Veränderungen feststellen.

Außer Quellungserscheinungen, die ja auch künstlich hervorgerufen und nicht mit voller Sicherheit als pathologiseh angesprochen werden können, wurden mehrfach im Mann-Alzheimerschen und im Bielschowskypräparat körnig zerfallene Achsenzylinder beobachtet (Taf. XIX, Fig. 50). Diese Körner hatten im Mann-Alzheimerpräparate bald eine blaue, bald eine rötliche Farbe. Außerdem wurden bei dieser Färbung häufig hellrot gefärbte Achsencylinder beobachtet, 
die außerdem auch morphologische Veränderungen, Auftreibungen, Schlängelungen, zuweilen körnige Struktur aufwiesen.

Während es vorkommen kann, daß auch normale Achsencylinder sich im Alzhei mer - Ma n nschen Präparate rot färben, zeigen dieselben gewöhnlich keine morphologischen Abweichungen von der Norm, dagegen zeigen, wie es Alzhei mer nachgewiesen hat, die degenerierten hellroten Achsencylinder deutliche Veränderungen in ihrer Struktur.

Was die Markscheide anbetrifft, so konnten in derselben schon bei der Mann-Alzheimerschen Färbung deutliche Veränderungen festgestellt werden. Es handelte sich um Auflösung des Markes in größere oder kleinere eosingefärbte Ringelchen und Schollen.

Im Weigertschen und im Spielmeyerschen Markscheidenpräparat konnten leichtere Ausfälle nicht konstatiert werden, dagegen

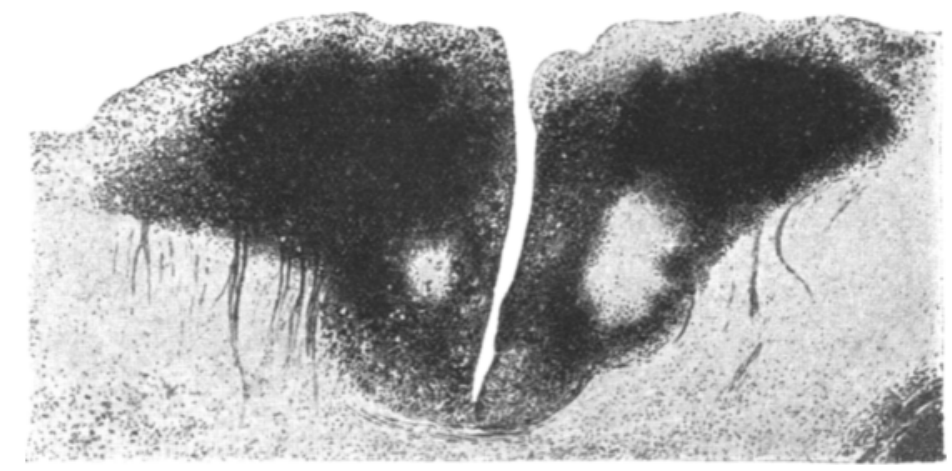

Fig. 3. Kaninchen XXIII. Spi elm eyersche Markscheidenmethode. Deutliche marklose Plaques in den Hintersträngen des Rückenmarks.

konnten in einem Falle mit der Spielmeyerschen Methode Markausfälle in Form von Plaques festgestellt werden. Es handelte sich um ein gravides Kaninchen, welches 35 Tage mit kleinen Dosen von Thyreoidin $(0,05$ täglich) gefüttert wurde. Das Kaninchen, welches von der Fütterung ein Gewicht von $3350 \mathrm{~g}$ zeigte, verlor binnen der 35 Tage $1200 \mathrm{~g}$, also $35 \%$ seines Gewichts. Es zeigte auch alle übrigen, für die Thyreotoxikose charakteristischen klinischen Erscheinungen.

Am 35. Tage wurde das Kaninchen getötet.

Im Rückenmark des Kaninchens wurden Verflüssigungserscheinungen an den Ganglienzellen, viele körnig zerfallenen Achsencylinder, zahlreiche Rosentalsche präamöboide Kerne, weniger zahlreiche, ausgesprochene, amöboide Gliazellen, vereinzelte Jacobsche Myeloklasten mit roten Körnern festgestellt. Der interessanteste Befund konnte aber im Spielmeyerschen Markscheidenpräparat erhoben werden. An einzelnen Stellen des Rückenmarks, sowohl an Quer-, als an 
Längsschnitten konnten in den Hintersträngen deutliche Plaques beobachtet werden (Textfig. 3, 4).

Da Spielmeyer in seiner Technik selbst darauf hinweist, daß zuweilen, wenn die Schnitte zu kurz im Spiritus geblieben sind, hier und da helle und schmutzig-blau gefärbte Flecke entstehen können, wurden frische Präparate dreimal hintereinander mit allen Kautelen angestellt. Das Ergebnis war immer dasselbe; an derselben Stelle konnten immer dieselben Plaques festgestellt werden. Auch der Vergleich dieser Präparate mit Mann - Alzheimerschen Präparaten aus derselben Region sprach dafür, daß es sich keineswegs um Kunstprodukte handelt. Das Mark war an denselben Stellen in feine Kügelchen und Brocken zerfallen, die das Eosin färbte, das Eisenhämatoxylin nicht mehr tingieren konnte. Die Gliazellen waren deutlich gewuchert.

Leider hatten wir aus dieser Region kein Chrommaterial; an Weigertschen und Spielmeyerschen Markscheidenpräparaten aus anderen Regionen konnten aber keine Plaques beobachtet werden. Scheinbar handelte es sich in diesem Falle um einen diskontinuierlichen, toxischen Markscheidenzerfall im Sinne Stransk ys. Möglich, daß in diesem Falle die Gravidität auch eine gewisse Rolle spielte. Jedenfalls würden die in diesem Falle erhaltenen Plaques für die Möglichkeit einer toxischen Entste-

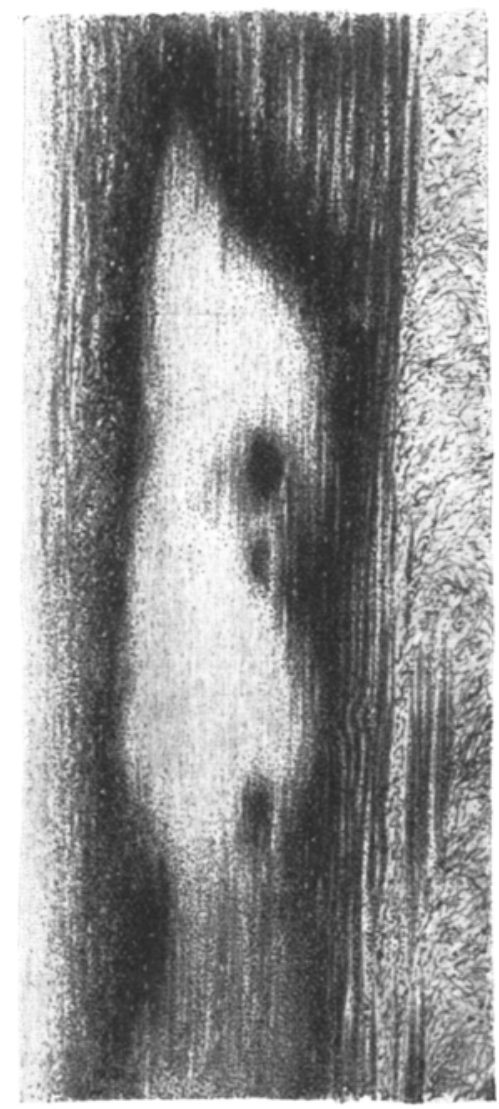

Fig, 4. Kaninchen XXIII. Längsschnitt des Rückenmarks. Spielmeyersche Markseheidenmethode. Große marklose Plaque im Hinterstrang. hung der multiplen Sklerose, deren Ätiologie bis jetzt noch so dunkel ist, sprechen (vgl. Flatau und Koelichen ${ }^{5}$ ).

Was das gliöse Gewebe der weißen Substanz anbetrifft, so ist es in allen Fällen von perakuter Thyreotoxikose schwer betroffen. Das Gliareticulum erscheint im Mann-Alzheimerschen Präparate verwaschen, gequollen, häufig körnig zerfallen. Die Gliazellen selbst sind in schweren Fällen sämtlich amöboid umgewandelt und zeigen alle für 
diese Umwandlung charakteristischen Eigenschaften, wie sie Rosental in exaktester Weise geschildert hat.

Der Kern wird klein, dunkel, homogen, zuweilen ist der Inhalt des Kerns lichtbrechend (Taf. XIX, Fig. 52). Das homogenisierte Plasma, welches in der grauen Substanz zumeist abgerundet erscheint, ist hier mehr in die Länge gezogen. Auch sieht man hier häufiger Zerfallerscheinungen in Form von feinerer oder gröberer Körnelung des Plasmas (Methylblaugranula Alzheimers) (Taf. XIX, Fig. 53-55). Zuweilen bilden sich im Plasma der amöboiden Zelle Vakuolen (Taf. XIX, Fig. 50, links unten, und Fig. 51). Manchmal sieht man, wie eine amöboide Gliazelle eine Nervenfaser umgibt (Taf. XIX, Fig. 51).

Bei den getöteten Kaninchen war die amöboide Umwandlung der Gliazellen weniger verbreitet, als bei den spontan zugrunde gegangenen, auch bei sofortiger, nach dem Tode erfolgter Fixierung des Materials schwächer ausgeprägt, als wenn die Fixierung einige Stunden nach dem Tode erfolgte. Es scheint hier der agonale Zustand, und, wie schon Rosental darauf hingewiesen hat, das Fortschreiten von während des Lebens begonnenen Verflüssigungs- und Abbauvorgängen die Bildung von amöboiden Gliazellen, bzw. den Zerfall derselben in Methylblaugranula zu begünstigen.

Außer der amöboiden Umwandlung der Gliazellen, die von Rosental wohl mit Recht als eine regressive Veränderung aufgefaßt wird, konnten in vielen, und zwar hauptsächlich in leichteren Fällen von Thyreotoxikose auch Wucherungserscheinungen an den Gliazellen beobachtet werden.

So konnten in Fällen, wo nur kleine Thyreoidindosen, oder größere Dosen, aber nur kurze Zeit eingeführt wurden, amöboide Gliazellen vermißt werden, während das Zellprotoplasma der Gliazellen, ebenso wie die faserigen Ausläufer derselben deutliche Wucherungserscheinungen aufwiesen.

Im Falle XXIII, in welchem deutliche plaqueartige Markscheidenausfälle festgestellt werden konnten, waren die Gliazellen in den Plaques deutlich gewuchert, die Zelleiber und die Kerne derselben waren größer, die Fasern dicker und länger, als im normalen Rückenmark.

In denselben Herden wurden vereinzelte Gliazellen, welche kleine rote Körner enthalten, die offenbar Produkte der Karyorrhexis darstellen, beobachtet. Diese Zellen hat Jacob in seiner eingehenden Studie über die sekundären Degenerationen als Myeloklasten bezeichnet.

An den Gefäßen der grauen und weißen Substanz des Rückenmarks konnten keine deutlichen Veränderungen erhoben werden. In den perakuten Fällen hatte man zuweilen den Eindruck, daß die Endothelzellen gewuchert sind, in den subakuten Fällen erschienen manchmal die Gefäßwandzellen geschrumpft und intensiv gefärbt, deutliche und 
allgemeine Alterationen der Gefäße konnten jedoch nicht festgestellt werden.

Blutungen und Infiltrationen der Gefäßscheiden waren in keinem einzigen Falle vorhanden.

An der Pia mater des Rückenmarks konnte, ebenso wie an den Gefäßen, nichts deutlich Pathologisches wahrgenommen werden.

Medulla oblongata, Pons und Ganglien des Mittelhirns.

In der Oblongata, im Pons Varoli und in den Ganglien des Mittelhirns konnten ähnliche Verflüssigungsvorgänge an den Ganglienzellen und amöboide Umwandlung der Gliazellen wie im Rückenmark festgestellt werden, doch waren dieselben nicht so hochgradig. Auch die Veränderungen der Markscheiden und Achsenzylinder waren hier schwächer ausgeprägt.

Hirnrinde.

Während die Ganglienzellen des Rückenmarks hauptsächlich Verflüssigungsvorgänge aufwiesen, zeigten die Ganglienzellen der Hirnrinde zumeist leichtere Veränderungen. Die färbbare Substanz des Zellkörpers war zumeist leidlich gut erhalten. Die protoplasmatischen Fortsätze waren zumeist weit gefärbt und häufig mit Körnern von färbbarer Substanz ausgefüllt; zuweilen konnte man korkzieherartige Fortsätze sehen (Taf. XVI, Fig. 14, 17). Trabantzellen mit gewuchertem Zelleibe schmiegten sich häufig eng an die Ganglienzellen hinan (Taf. XVI, Fig. 15), zuweilen war der Ganglienzellkörper zwischen den Trabantzellen eng zusammengepreßt und dadurch eingekerbt und in die Länge gezogen (Taf. XVI, Fig. 17). Die Trabantzellen zeigten mitunter amöboide Umwandlung (Taf. XVI, Fig. 13a). Der Kern der Ganglienzellen war blaß, die Membran aufgelöst (Taf. XVI, Fig. 13), zuweilen war der Kern intensiv blau gefärbt und die Membran erhalten (Fig. 15, 16).

Im allgemeinen waren die toxischen Veränderungen in der Hirnrinde viel schwächer ausgeprägt, als im Rückenmark.

Kleinhirn.

Erhebliche Alterationen konnten bei mit Thyreoidin vergifteten Kaninchen im Kleinhirn festgestellt werden.

Die Ganglienzellen des Nucleus dentatus (Taf. XV, Fig. 10) und der übrigen Kerne des Kleinhirns waren stark verändert und zumeist in verschiedenen Stadien der Verflüssigung begriffen.

Die Kleinhirnrinde zeigte in ihren verschiedenen Schichten verschiedenartige Alterationen. In der Körnerschicht konnte weder Lichtung, noch deutliche Veränderung der Struktur der Körnerzellen wahrgenom- 
men werden. Dagegen die großen Zellen der Körnerschicht (die sog. Golgischen Zellen) wiesen deutliche Veränderungen auf und waren in verschiedenen Stadien des Zerfalls der färbbaren Substanz begriffen. Zuweilen blieb von so einer Zelle nur ein Schatten zurück (Taf. XVII, Fig. 22, die Zelle liegt unterhalb einer amöboiden Gliazelle a). In der Schicht der P urki njeschen Zellen waren fast alle Ganglienzellen schwer erkrankt.

Die färbbare Substanz des Zellkörpers der Purkinjezellen war in verschiedenen Stadien des Zerfalls begriffen; von einzelnen Zellen ist nur ein blasser kernloser Schatten zurückgeblieben, welcher von gewucherten Gliazellen bedeckt war (Fig. 22p).

In der Molekularschicht waren die größeren Ganglienzellen, die sog. Korbzellen leidlich gut erhalten; nur waren sie häufig von zahlreichen Gliakernen umgeben (Fig. 22g). An einzelnen Ganglienzellen der Molekularschicht konnten zuweilen 10-20 Gliakerne aufgezählt werden. Es scheint sich aber nicht um eine pathologische Gliawucherung zu handeln, weil ähnliche Anhäufung von Gliakernen um diese Zellen auch im Kleinhirn gesunder Kaninchen und bei verschiedenartigen (normalem und pathologischem) Menschenmaterial festgestellt werden konnte.

Offenbar handelt es sich um eine physiologische Anhäufung von Gliakernen um gewisse Nervenzellen, eine Tatsache, die ein gewisses histologisches Interesse bietet, aber weder mit Neurophagie, noch speziell mit der Thyreoidinvergiftung was Gemeinschaftliches hat.

Eine ganz eigentümliche Veränderung konnte in den kleineren Ganglienzellen der Molekularschicht beobachtet werden. Da diese Veränderung meines Wissens bisher noch nicht beschrieben worden ist, möchten wir auf dieselbe ausführlicher eingehen.

Zwischen normalen kleineren Ganglienzellen (Fig. $22 \mathrm{k}$ ) der Molekularschicht, die weder im Kern, noch im Plasmaleib und Fortsätzen deutliche Veränderungen aufwiesen (außer vereinzelten lipoiden Körnchen, die zuweilen in den Fortsätzen beobachtet werden konnten, siehe Fig. 22 Molekularschicht links) wurden öfters Zellen beobachtet, die auf den ersten Blick sehr an Plasmazellen erinnerten. Derselbe dunkle Kern mit radspeichartig an der Peripherie angeordneten Chromatinkörnern, derselbe helle Hof um den Kern und leicht metachromatische Färbung des erhaltenen Zellplasmas.

Das nähere Studium dieser Zellveränderung zeigte, daß es sich weder um Plasmazellen, noch um veränderte Gliazellen, sondern daß es sich um eine eigentümliche Degenerationsform der kleinen Ganglienzellen der Molekularschichte handelt. Bei Durchsicht mehrerer Präparate konnten verschiedene Stadien dieser Veränderung festgestellt werden.

Zunächst scheint der Kern ergriffen zu werden. Der normalerweise ziemlich große, im Thioninpräparate hellblaue Kern (Taf. XVII, Fig. 22 k, 
Fig. 34, 35) wird kleiner und zeigt eine homogen dunkle Färbung, die Membran löst sich auf. Statt deren sehen wir an der Peripherie des Kerns radspeichartig angeordnete Chromatinkörner, vereinzelte Körner sind zuweilen auch im Inneren des Karioplasmas sichtbar (Fig. 22x, 39), der ganze Kern wird allmählich einer Maulbeere ähnlich. Das Plasma des Zellkörpers färbt sich immer blasser und beginnt sich zunächst um den Kern herum aufzulösen, so daß sich ein heller Hof bildet (Fig. 22 x, 26, 27, 28, 31, 39). In Anfangsstadien ist noch deutlich der Spitzenfortsatz sichtbar (Fig. 23, 26, 28, 39), allmählich löst sich das Plasma der Fortsätze und des Körpers mehr und mehr auf. Schließlich bleibt von der ganzen Zelle nur ein dunkelblauer, vielfach eingekerbter (Fig. 23a, $24,26,36,37$ ), maulbeerartiger Kern mit intensiv blau gefärbten Kernkörperchen und ringartigem Plasmasaum zurück (Fig. 23a, 38, 36, 37).

In diesem Stadium sind die Zellen vielfach den Plasmazellen sehr ähnlich.

Es interessierte uns zunächst, ob auch beim Menschen diese Zellveränderung vorkommt; es wurden zu diesem Zwecke zahlreiche Kleinhirnpräparate durchmustert. Bei Paralyse, seniler Demenz, Alzhei merscher Krankheit, Dementia praecox, Epilepsie und einer Reihe anderer Erkrankungen des Zentralnervensystems konnten wir keine einzige derartige Zelle auffinden, dagegen haben wir in einem Falle von Meningitis tuberculosa mit Tuberkeln im Kleinhirn und in einem Falle von Gliosarkom des Kleinhirns in der Nähe der Neubildung genau dieselben Zellen in der Molekularschicht des Kleinhirns beobachten können. Fig. 23-25, 36-39 zeigen verschiedene Stadien dieser Zellerkrankung in einem Falle von Gliosarcoma cerebelli beim Menschen.

Auch bei Hunden konnten wir dieselbe Zelldegeneration und ebenfalls in der Molekularschicht des Kleinhirns nachweisen, und zwar in mehreren Fällen von experimenteller, durch Pneumokokken erzeugter Cerebrospinalmeningitis*).

In der normalen Kleinhirnrinde konnte weder beim Kaninchen, noch beim Menschen und Hunde je eine einzige ähnliche Zelle beobachtet werden.

Es handelt sich offenbar um eine eigenartige Degenerationsform gewisser Ganglienzellen, und die nächste Frage war, ob diese Degeneration ausschließlich die Ganglienzellen der Molekularschicht des Kleinhirns ergreift.

Es wurde das ganze zentrale Nervensystem von einigen Kaninchen, die an akuter Thyreotoxikose zugrunde gegangen sind, speziell in dieser Hinsicht durchsichtet. Es erwies sich, daß diese Veränderung,

*) Diese Präparate wurden mir von Herren Dr. Flatau und Dr. Handelsman in liebenswürdiger Weise zur Verfügung gestellt, wofür ich ihnen meinen herzlichsten Dank sage. 
außer der Molekularschicht des Kleinhirns, noch die Ganglienzellen des Tuberculum acusticum und die kleinen Ganglienzellen der Hinterhörner des Rückenmarks ergreift. Sonst konnten wir nirgend im zentralen Nervensystem der mit Thyreoidin vergifteten Kaninchen ähnlich veränderte Ganglienzellen auffinden.

Diese Tatsache, daß nur ganz bestimmte Ganglienzellen in genau beschränkten Bezirken des Zentralnervensystems an dieser Degenerationsform zugrunde gehen, während andere in der nächsten Nachbarschaft sich befindende Ganglienzellen ganz andere Degenerationsformen aufweisen, scheint von erheblichem biologischem Interesse zu sein.

In meinen Arbeiten über die senile Demenz ${ }^{10}$ ) und über die Alzheimersche Krankheit ${ }^{12}$ ) versuchte ich ebenfalls genauer auf lokalisatorische Verhältnisse einzugehen, und es gelang mir nachzuweisen, daß bei beiden diesen Krankheiten die Pyramiden des Ammonhorns eine Degenerationsform aufweisen, die sonst nirgend im Zentralnervensystem zu finden ist. Diese Ganglienzellveränderung, die in beiden diesen Arbeiten genau geschildert worden ist, wurde in der ersten Arbeit grobkörnige, in der zweiten körnig-vakuoläre Degeneration genannt.

Wir sehen also, daß sowohl bei Erkrankungen des menschlichen Nervensystems wie bei experimentell bei Tieren erzeugten Erkrankungen dieselbe Noxe an verschiedenen Stellen des Nervensystems ganz verschiedenartige Ganglienzellveränderungen erzeugen kann, daß gewisse Degenerationsformen zuweilen nur ausschließlich in bestimmten Ganglienzellgruppen vorkommen können.

Diese Tatsachen scheinen dafür zu sprechen, daß nicht nur Zellen verschiedener Organe, sondern Zellen, die im selben Organ und sogar in derselben Zellschicht nebeneinander sich befinden (wie z. B. die größeren und kleineren Ganglienzellen der Molekularschicht der Kleinhirnrinde) höchst wahrscheinlich einen ganz anderen Bau und andere Funktionen besitzen, sonst würden sie nicht in so verschiedener Weise auf dasselbe Gift reagieren.

Während es sich erwiesen hat, daß es für verschiedene Vergiftungen keine spezifischen Zellerkrankungen gibt, wird es sich vielleicht nachweisen lassen, daß es für gewisse Zellarten mehr oder weniger spezifische Erkrankungen gibt.

Außer dem zentralen Nervensystem wurden bei den mit Thyreoidin vergifteten Kaninchen die interspinalen und die sympathischen Ganglien untersucht. Besonders haben uns die letzteren interessiert, weil ja bis jetzt noch öfters die Based ow sche Krankheit auf eine primäre Affektion des sympathischen Nervensystems zurückgeführt wird und die Veränderungen der Schilddrüse als sekundär betrachtet werden. So scheint es von Interesse zu sein, daß durch Thyreoidinfütterung in den sympathischen Ganglien schwere Alterationen hervorgerufen werden können. 
Fig. 40-46 illustrieren den allmählichen Zerfall der Ganglienzellen des Grenzsympathicus mit Vakuolisierung des Plasmaleibes (Fig. 41 bis 43), Auflösung der färbbaren Substanz und schließlich Wucherung der Kapselzellen auf den Trümmern der Ganglienzelle (Fig. 42, 45, 46).

Ganz ähnliche Veränderungen konnten auch in den Spinalganglienzellen festgestellt werden. An der Fig. 47 sehen wir noch eine fast normale Spinalganglienzelle, nur der Kern hebt sich nicht scharf genug vom Zellkörper ab; in der nächsten Figur (48) zeigt der Kern deutliche Degenerationserscheinungen, der Plasmaleib ist im Zerfall und Vakuolisierung begriffen; an der nächsten Figur (49) sehen wir zwei fast vollständig zerfallene Spinalganglienzellen mit hineinwuchernden Kapselzellen.

Wenn wir nun die histologischen Veränderungen, die wir bei experimenteller Thyreotoxikose im Nervensystem des Kaninchens feststellen konnten, zusammenfassen wollen, so können wir wohl folgendes sagen.

Sowohl im zentralen Nervensystem, wie in den spinalen und sympathischen Ganglien konnten schwere degenerative Veränderungen festgestellt werden. Die bedeutendsten Störungen sind im Rückenmark, sowie auch in den spinalen und sympathischen Ganglien beobachtet worden, auch im verlängerten Mark, Pons, Ganglien des Mittelhirns und im Kleinhirn konnten bedeutende Veränderungen nachgewiesen werden, dagegen zeigte die Hirnrinde weniger intensive, obwohl ebenfalls deutliche Alterationen.

Die Ganglienzellen des Rückenmarks und des verlängerten Markes wiesen zumeist verschiedene Typen und Stadien des Verflüssigungsprozesses auf, Markscheiden und Achsenzylinder zeigten zuweilen deutliche Veränderungen, in einem Falle konnte im Rückenmark diskontinuierlicher Zerfall von Markscheiden in Form von ziemlich großen marklosen Plaques, welche dem Bilde nach denjenigen bei multipler Sklerose ähnlich erschienen, festgestellt werden. Die Gliazellen waren in allen schwereren Fällen amöboid umgewandelt, in den leichteren Fällen konnten an den Gliazellen Wucherungserscheinungen nachgewiesen werden.

Im Kleinhirn konnten erhebliche degenerative Veränderungen beobachtet werden, besonders beachtenswert ist die eigenartige Verände: rung der kleineren Ganglienzellen der Molekularschicht, welche außerhalb des Kleinhirns nur noch in den Ganglienzellen des Tuberculum acusticum und der Hinterhörner des Rückenmarks festgestellt werden konnte.

An den Gefäßen des zentralen Nervensystems konnten nirgend deutliche Veränderungen festgestellt werden. Die Ganglienzellen der sympathischen und spinalen Ganglien gingen ebenfalls zugrunde, wobei die Kapselzellen deutliche Wucherungserscheinungen zeigten. 
Bei unseren Kontrollversuchen mit tierischem Eiweiß konnten wir keine toxischen Veränderungen im Nervensystem feststellen, bei den Versuchen mit Jod, wobei 500 mal größere Dosen von Jod, als es im Thyreoidin enthalten ist*), den Kaninchen eingeführt wurden, konnten in Zentralnervensystem nur ganz leichte toxische Veränderungen nachgewiesen werden, die in keiner Weise an die Veränderungen bei Thyreotoxikose erinnerten. Es unterliegt also keinem Zweifel, daß die Veränderungen im Nervensystem weder auf eine Intoxikation mit heterogenem Eiweiß, noch auf Jodvergiftung zurückzuführen ist.

Damit sei keineswegs gesagt, daß die von uns festgestellten Veränderungen für die Thyreoidinvergiftung spezifisch sind. Erst in der jüngsten Zeit wurden bei Kaninchen ähnliche, wenn auch schwächer ausgeprägte Veränderungen von Rosental durch Guanidinvergiftung erzeugt.

Wir haben selber durch intravenöse Injektion von Tetanustoxin bei Kaninchen ganz ähnliche Veränderungen im Nervensystem erzeugen können, dieselben aber weniger intensiv, die Verflüssigungsvorgänge an den Ganglienzellen nicht so hochgradig, die amöboide Umwandlung cler Gliazellen nicht so allgemein, wie bei Kaninchen die an akuter Thyreotoxikose zugrunde gingen.

Die in dieser Mitteilung beschriebenen Experimente zeigen jedenfalls zum ersten Male, da $\beta$ durch Einverleibung von Blutdrüsenextrakten schwere degenerative Veränderungen sowohl im zentralen Nervensystem, wie auch in den sympathischen und spinalen Ganglien entstehen können.

Unsere Untersuchungen sind noch nicht abgeschlossen, es werden jetzt weitere Untersuchungen mit Thyreoidin an Affen und mit Pituitrin an Kaninchen vorgenommen. In der nächsten Mitteilung wird ebenfalls auf die Abbauverhältnisse und auf die topographische Verteilung der Veränderungen im zentralen und peripheren Nervensystem näher eingegangen werden.

In clieser Mitteilung kam es uns hauptsächlich darauf an, zu zeigen, da $\beta$

1. bei Kaninchen durch Einführung von Thyreoidin schwere toxische, mikroskopischnachweisbareVeränderungen im Nervensystem erzeugt werden können,

2. daß bei längerer Darreichung von Thyreoidin sogar bei sehr kleinen Dosen (0,015 pro $1 \mathrm{~kg}$ täglich), die etwa den medikamentösen Dosen bei Menschen entsprechen, bei Kaninchen sämtliche klinischen und histologischen Erscheinungen der Thyreotoxikose festgestellt werden können,

3. daß Hunde und Kaninchen auf das Thyreoidin in verschiedener Weise reagieren.

*) Der Jodgehalt normaler Schilddrüsen beträgt $0,3-0,9 \mathrm{mg}$ auf $1 \mathrm{~g}$ Trockensubstanz (Wagner v. Jauregg und Bayer). 
Histologische Veränderungen im Nervensystem bei Thyreotoxikose. 295

Ủbersicht über sämtliche Versuchstiere.

\begin{tabular}{|c|c|c|c|c|c|c|}
\hline$=-=$ & $\begin{array}{c}\text { Gewicht } \\
2 \\
\mathrm{~g}\end{array}$ & $\begin{array}{l}\text { Eingefürte Menge } \\
\text { von Thyreoidin }\end{array}$ & $\begin{array}{c}\text { Ge- } \\
\text { storben } \\
\text { Tag }\end{array}$ & $\begin{array}{l}\text { Ge- } \\
\text { tötet } \\
\text { Tag }\end{array}$ & $\begin{array}{c}\text { Gewicht } \\
\text { nach } \\
\text { dem } \\
\text { Tode } \\
\text { g } \\
\end{array}$ & Bemerkungen \\
\hline \multicolumn{7}{|l|}{ Kaninchen } \\
\hline 1 & 1020 & $\begin{array}{l}8 \times 0,3+6 \times 1,0 \\
=8,4 \text { per os }\end{array}$ & 16 & & 660 & \\
\hline 2 & 1000 & $\begin{array}{l}7 \times 0,3+4 \times 1,0 \\
=6,1 \text { per os }\end{array}$ & 12 & & 720 & \\
\hline 3 & 800 & $7 \times 1,0$ per os & 7 & & 575 & \\
\hline 4 & 1130 & $10 \times 1,0$ per os & 10 & & 670 & \\
\hline 5 & 1900 & $11 \times 1,5$ per os & 11 & & 1100 & \\
\hline 6 & 1850 & $\begin{array}{c}1 \times 2,0+9 \times 3,0 \\
=29,0 \text { per os }\end{array}$ & 12 & & 1030 & \\
\hline 7 & 2750 & $\begin{array}{l}2 \times 3,0+8 \times 0,5 \\
+15 \times 1,0 \\
=25,0 \text { per os }\end{array}$ & 35 & & 1800 & \\
\hline 8 & 1700 & $\begin{array}{l}2 \times 3,0+6 \times 0,5 \\
=9,0 \text { per os }\end{array}$ & & 9 & 1450 & Exophthalmus \\
\hline 9 & 1700 & $\begin{array}{c}1 \times 1,0+6 \times 0,5 \\
=4,0 \text { per os }\end{array}$ & & 8 & 1400 & \\
\hline 10 & 3400 & $2 \times 3,0=6,0$ per os & & 4 & 2970 & \\
\hline 11 & 2020 & $2 \times 3,0=6,0$ per os & & 4 & 1700 & \\
\hline 12 & 3930 & $5 \times 0,1=0,5$ per 08 & & 6 & 3850 & \\
\hline 13 & 2720 & $5 \times 0,1=0,5$ per os & & 6 & 2580 & \\
\hline 14 & 2000 & $5 \times 0,1=0,5$ per os & & 6 & 1650 & \\
\hline 15 & 2680 & $4 \times 0,1=0,4$ per os & & 5 & 2250 & \\
\hline 16 & 1830 & $\begin{array}{l}1 \times 2,0+3 \times 3,0 \\
=11 \mathrm{~g} \text { per os }\end{array}$ & & 5 & 1500 & \\
\hline 17 & 2450 & $4 \times 3,0=12,0$ & & 5 & 1950 & \\
\hline 18 & 2850 & $22 \times 0,1=2,2$ per os & 27 & & 1400 & \\
\hline 19 & 2350 & $18 \times 0,1=1,8$ per os & 22 & & 1550 & $\begin{array}{c}\text { Vor dem Tode tonische } \\
\text { Zuckungen }\end{array}$ \\
\hline 20 & 2000 & $19 \times 0,1=1,9$ per os & & 23 & 1450 & \\
\hline 21 & 2400 & $3 \times 3,0=9,0$ peros & & 4 & 2050 & \\
\hline 22 & 1600 & $\begin{array}{c}25 \times 0,05=1,25 \\
\text { per os }\end{array}$ & 36 & & 1050 & \\
\hline 23 & 3350 & $\begin{array}{c}25 \times 0,05=1,25 \\
\text { per os }\end{array}$ & & 36 & 2380 & \\
\hline 24 & 2150 & $\begin{array}{r}10 \times 2,0=20,0 \\
\text { per os }\end{array}$ & 18 & & 1350 & \\
\hline 25 & 2200 & $6 \times 2,0$ & 7 & & 1320 & \\
\hline 26 & 1900 & $\begin{array}{c}4 \times 0,3+2 \times 0,5 \\
=2,2 \text { subcutan }\end{array}$ & 18 & & 1300 & \\
\hline 27 & 2000 & $\begin{array}{c}3 \times 0,5+2 \times 0,3 \\
=2,1 \text { subcutan }\end{array}$ & 10 & & 1550 & \\
\hline 28 & 1800 & $\begin{array}{l}2 \times 0,5+4 \times 0,25 \\
=2,0 \text { subcutan }\end{array}$ & 26 & & 1275 & \\
\hline
\end{tabular}


(Fortsetzung.)

\begin{tabular}{|c|c|c|c|c|c|c|}
\hline Nr. & Gewicht & $\begin{array}{c}\text { Eingeführte Menge } \\
\text { von Thyreoidin } \\
\mathrm{g}\end{array}$ & $\begin{array}{c}\text { Ge- } \\
\text { storben } \\
\text { Tag }\end{array}$ & $\begin{array}{c}\text { Ge- } \\
\text { tötet } \\
\text { Tag }\end{array}$ & $\left|\begin{array}{c}\text { Gewicht } \\
\text { nach } \\
\text { dem } \\
\text { Tode } \\
\mathrm{g}\end{array}\right|$ & Bemerkungen \\
\hline Kaninchen & & & & & & \\
\hline 29 & $\begin{array}{c}3050 \\
19.1 I .13\end{array}$ & $\begin{array}{l}6 \times 5,0 \text { Basedow- } \\
\text { schilddruse per os }\end{array}$ & - & - & $\begin{array}{c}2550 \\
\text { 28. II. } 13\end{array}$ & \\
\hline 30 & 2220 & $\begin{array}{l}3 \times 0,2+9 \times 0,5 \\
+6 \times 1,0+4 \\
\times 1,2+6 \times 2,0 \\
=27,9 \text { Kalii jodati } \\
\text { subcutan (teilweise } \\
\text { in } 10 \text { proz. teil- } \\
\text { weise in } 20 \text { proz. } \\
\text { Lösung) }\end{array}$ & 65 & & 1850 & \\
\hline 31 & 2250 & $\begin{array}{l}3 \times 0,1+4 \times 0,5 \\
+2 \times 1,0=4,3 \\
\text { Kalii jodati sub- } \\
\text { cutan in } 20 \text { proz. } \\
\text { Lösung }\end{array}$ & 12 & & 1550 & \\
\hline 32 & 1930 & $\begin{array}{l}4 \times 0,3+2 \times 0,65 \\
+4 \times 1,0=6,5 \\
\text { Kalii jodati sub- } \\
\text { cutan in } 20 \text { proz. } \\
\text { Lösung }\end{array}$ & 18 & & 1300 & \\
\hline 33 & 2050 & $\begin{array}{l}17 \times 1+8 \times 2 \\
\text { Eiereiweiß per os }\end{array}$ & & 47 & 2000 & \\
\hline 34 & 2450 & $\begin{array}{l}5 \times 1 \text { Eiereiweiß } \\
\text { per os }\end{array}$ & & 5 & 2440 & \\
\hline 35 & 2050 & $\begin{array}{l}17 \times 1+6 \times 2 \text { per os } \\
\quad+1 \text { subcutan } \\
\quad+2 \text { subcutan }\end{array}$ & 49 & & 2000 & $\begin{array}{l}\text { Am nächsten Tage nach } \\
\text { der subcutanen Injek- } \\
\text { tion von } 2 \text { Eiern tot } \\
\text { gefunden. (Auaphy- } \\
\text { laxie?) }\end{array}$ \\
\hline 36 & 1780 & $\begin{array}{l}25 \times 1 \text { Teelöffel Lie- } \\
\text { bigs Fleischextrakt } \\
\text { per os }\end{array}$ & & 39 & 1800 & \\
\hline 37 & 2250 & $\begin{array}{l}20 \times 25 \mathrm{~g}=500 \mathrm{~g} \\
\text { Fleisch per os }\end{array}$ & & 28 & 2400 & \\
\hline 38 & 2150 & $\begin{array}{c}20 \times 35 \mathrm{~g}=700 \mathrm{~g} \\
\text { Fleisch per os }\end{array}$ & & 28 & 2300 & \\
\hline 39 & 2250 & $\begin{array}{c}2 \times 1 \text { Eiereiweiß } \\
\text { per os }+4 \times 1 \\
\text { Eier subcutan }\end{array}$ & & 15 & 2200 & \\
\hline 40 & 2950 & $\begin{array}{l}3 \times 2 \text { Eier per os } \\
+5 \times 1 \text { subcutan }\end{array}$ & & 17 & 2850 & \\
\hline 41 & 2550 & $\begin{array}{l}9 \times 25 \mathrm{~g}=225 \mathrm{~g} \\
\text { Schweinsleber } \\
\text { per os }\end{array}$ & & 18 & 2700 & \\
\hline 42 & 1750 & $\begin{array}{l}9 \times 30 \mathrm{~g}=270 \mathrm{~g} \\
\text { Schweinsleber } \\
\text { per os }\end{array}$ & & 18 & 2000 & \\
\hline
\end{tabular}


Histologische Veränderungen im Nervensystem bei Thyreotoxikose. 297

(Fortsetzung.)

\begin{tabular}{|c|c|c|c|c|c|c|}
\hline Nr. & $\begin{array}{c}\text { Gewicht } \\
\mathbf{g} \\
\end{array}$ & $\begin{array}{c}\text { Eingefinrte Menge } \\
\text { von Thyreoidin } \\
\end{array}$ & $\left|\begin{array}{c}\text { Ge- } \\
\text { storben } \\
\text { Tag }\end{array}\right|$ & $\begin{array}{l}\text { Ge- } \\
\text { tőtet } \\
\text { Tag }\end{array}$ & $\left|\begin{array}{c}\text { Gewicht } \\
\text { nach } \\
\text { dem } \\
\text { Tode } \\
\text { g }\end{array}\right|$ & Bemerkungen \\
\hline \multicolumn{7}{|l|}{ Hund } \\
\hline 1 & 8500 & $\begin{array}{l}2 \times 1,5+5 \times 3,0 \\
+2 \times 6,0+7 \\
\times 12,0+1 \times 20,0 \\
+9 \times 30,0+3 \\
\times 50,0+2 \times 100,0 \\
=754 \mathrm{~g} \text { Thyreoidin } \\
\text { per os }\end{array}$ & 37 & & 5250 & \\
\hline 2 & 3900 & $\begin{array}{l}1 \times 6,0+1 \times 10,0 \\
+2 \times 15,0+2 \\
\times 20,0+2 \times 30,0 \\
=146 \mathrm{~g} \text { Thyreoidin } \\
\text { per os }\end{array}$ & & 26 & 3150 & \\
\hline 3 & 5200 & $\begin{array}{l}2 \times 6,0+1 \times 10,0 \\
+3 \times 15,0+1 \\
\times 25,0=92 \mathrm{~g} \\
\text { Thyreoidin per os }\end{array}$ & 10 & & 5030 & Plötzlicher Tod \\
\hline 4 & 5000 & $\begin{array}{l}2 \times 6,0+1 \times 10,0 \\
+3 \times 15,0+2 \\
\times 25,0=107 \mathrm{~g} \\
\text { Thyreoidin per os }\end{array}$ & & 20 & 3800 & \\
\hline 5 & 5300 & $\begin{array}{l}2 \times 1,0 \text { Thyreoidin } \\
\text { subcutan }\end{array}$ & & & & $\begin{array}{l}\text { Keine klinischen } \\
\text { Erscheinungen }\end{array}$ \\
\hline 6 & 5800 & $\begin{array}{l}3 \times 1,0 \text { Thyreoidin } \\
\text { subcutan }\end{array}$ & & & & $\begin{array}{l}11 \text { Wochen nach der letzten } \\
\text { Injektion konnten Haar- } \\
\text { ausfall am Schwelf und } \\
\text { gymmetrische nekrotische } \\
\text { Wunden an beiden Ohren } \\
\text { festgestellt werden. Ge- } \\
\text { wicht } 5300 \mathrm{~g} \text {. }\end{array}$ \\
\hline 7 & 3800 & $\begin{array}{l}1 \times 4,0 \text { Thyreoidin } \\
\text { subcutan }\end{array}$ & 85 & & 2700 & $\begin{array}{l}\text { Schwere trophische Verän- } \\
\text { derungen am Schweif und } \\
\text { Ohren (siehe Tafel XX, } \\
\text { Textflg. 1, 2). }\end{array}$ \\
\hline 8 & 4750 & $\begin{array}{l}1 \times 4,0 \text { Thyreoidin } \\
\text { subeutan }\end{array}$ & 155 & & 4450 & $\begin{array}{l}\text { Keine klinischen Erschei- } \\
\text { nungen, plötzlicher Tod. }\end{array}$ \\
\hline 9 & 3250 & $\begin{array}{l}1 \times 50 \mathrm{~g} \text { Basedow- } \\
\text { schildrüse per os }\end{array}$ & & & & $\begin{array}{l}\text { Keine klinischen Wrschei- } \\
\text { nungen. }\end{array}$ \\
\hline
\end{tabular}

\section{Literaturverzeichnis.}

1. Alzheimer, Beiträge zur Kenntnis der pathologischen Neurologie und ihrer Beziehungen zu den Abbauvorgängen im Nervengewebe. Nissl-Alzheimers histol. u. histopathol. Árb. üb. d. Großhirnrinde 3, H. 3.

2. Biedl, Innere Sekretion. 2. Auflage. 1913.

3. Birsch-Hirschfeld und Nobuo-Inouye, Experimentelle Untersuchun. gen über die Pathogenese der Thyreoidin-Amblyopie. Archiv f. Ophthalmol. 61. 
4. Erb ju n., Über Gehirnblutungen beim Kaninchen nach Adrenalininjektion. Beiträge z. pathol. Anat. u. z. allg. Pathol. \%, Supplement. Festschrift f. Arnold. 1905 .

5. Flatau und Koelichen, Über die multiple Sklerose. Archiv f. mikr. Anat. y8. 1911. Waldeyers Festschrift.

6. Klose, Lampe und Liesegang, Die Basedowsche Krankheit, eine chirurgisch-experimentelle und biologische Studie. Beiträge z. klin. Chir. $\gamma \gamma$, H. 3.

7. Rosental, St., Experimentelle Studien über amöboide Umwandlung der Neuroglia. Nissl-Alzheimers histol. u. histopathol. Arb. üb. d. Großhirnrinde 6, H. 1 .

8. Shima, Zur Frage der nach Adrenalinwirkung auftretenden Veränderungen des Centralnervensystems des Kaninchens. Neurol. Zentralbl. 1908.

9. - Experimentelle Untersuchungen über die Wirkung des Adrenalins auf das Zentralnervensystem des Kaninchens. Obersteiners Arb. 14.

10. Simchowicz, Histologische Studien über die senile Demenz. Nissl-Alzheimers histol. u. histopathol. Arb. üb. d. Großhirnrinde 4, H. 2.

11. - Etudes expérimentales sur les altérations du système nerveux, dependant des glandes à sécrétion interne. Compt. rend. de la Soc. scientifique de Varsovie, 5 Année, fasc. 9.

12. - La maladie d'Alzheimer et son rapport avec la démence sénile. L'Encóphale 1914, 3.

13. S pielme yer, Technik der mikroskopischen Untersuchung des Nervensystems. 2. Auflage. 1914.

14. Wagner v. Ja uregg und Gustav Ba yer, Lehrbuch der Organotherapie 1914. 


\section{Erklärung der Tafeln $\mathbf{X V}-\mathbf{X X}$.}

\section{Tafel XV.}

Alkoholfixierung. Thioninfärbung in Celloidin eingebetteter Schnitte. Vergrößerung: Fig. 1, 3, 6, 7-Zeiß, Ölimmersion, Ap. 1,30, Okul. 3. Fig. 2-Voigtländer, Immersion, Okul. 4. Fig. 4, 8, 10, 11, 12 - Zeiß, Ölimmersion Ap. 1,30, Okul. 2. Fig. 5-9 - Leitz, Immersion 1/12, Okul. 1.

Fig. 1. Kaninchen Nr. 2. Ganglienzelle aus den Vorderhörnern des Rückenmarks. Vom Zellkörper blieb kaum ein Saum zurück; der Rest ist mit Gliazellen ausgefüllt; Nisslschollen sind noch an der Basis und längs des geschwollenen und von amöboiden Gliazellen umgebenen protoplasmatischen Fortsatzes zu sehen.

a. Amöboide Gliazellen.

ep. Epithelioide Gliazellen.

eap. Ein der degenerierten Ganglienzelle anliegendes Capillargefäß.

Fig. 2, 3, 7. Kaninchen Nr. 2. Ganglienzellen aus den Vorderhörnern des Rücken. marks. Verschiedene Stadien des Zerfalls der färbbaren Substanz. Fig. 7 die Nisslschollen sind nur noch plaqueweise erhalten. Fig. 2. Der Zellkörper ist aufgedunsen, geschwellt und abgerundet; weder Kern, noch Fortsätze vorhanden; Nisslschollen nur noch in Gestalt von kleinen Plaques zurückgeblieben; Fig. 3. Nisslschollen blaß, kaum färbbar; der Ganglienzelle liegen 5 Gliazellen an.

Fig. 4. Kaninchen Nr. 6. Längsschnitt des Rückenmarks. Zelle aus den Vorderhörnern mit weit fortgeschrittenem Verflüssigungsprozeß. Die ganze Zelle erinnert an einen auf Löschpapier zerfließenden Tintenfleck. Im Innern der Zelle ist ein kleiner dunkler Kern zu sehen (K); rings um die Zelle - einige kleine pyknotische Gliakerne (G).

Fig. 5, 6. Kaninchen Nr. 2. Ganglienzelle aus den Vorderhörnern mit der Nisslschen , schweren Zellerkrankung“. Die diese Degeneration kennzeich. nenden Veränderungen im Plasma und im Kern; perinucleärer Plasma. zerfall (Fig. 6).

Fig 8. Kaninchen Nr. 2. Ganglienzelle aus den Seitenbörnern des Rückenmarks mit Vakuolen im Plasma.

Fig. 9. Ganglienzelle aus den Hinterhörnern des Rückenmarks; Körnchen der färbbaren Substanz sind im Dendrit auf großer Strecke zu verfolgen; dem Dendrit liegt eine amöboide Gliazelle an (a).

Fig. 10. Kaninchen Nr. 6. Ganglienzelle aus dem Nucle us dentatus cerebelli im Verflüssigungsprozeß begriffen. Die in Form von Bälkchen gelagerten basophile Körnchen sind an der Zellspitze besonders dicht zusammengehäuft.

Fig. 11. Kaninchen Nr. 6. Ganglienzelle aus dem Seitenhorn des Rückenmarks. Initialstadium der Nisslschen schweren Zellerkrankung. Die Zelle ist leicht angeschwollen; das Zellprotoplasma und die Fortsätze sind mit feinen Körnchen ausgefüllt. Der Kern beginnt seine Konturen zu verlieren und die Normalfarbe zu wechseln. 
Fig. 12. Kaninchen Nr. 6. Protoplasmatischer Fortsatz einer Ganglienzelle aus den Vorderhörnern, mit Körnchen gefüllt, welche stellenweise dicke, intensiv gefärbte Inseln bilden (V).

\section{Tafel XVI.}

Alkoholfixierung. Thioninfärbung in Celloidin eingebetteter Schnitte. Vergrößerung: Fig. 13 - Zeiß, Immersion, Okul. 3. Fig. 14-17 - Leitz, Immersion, Okul. 3. Fig. 18-2l - Zeiß, Immersion, Okul. 3.

Fig. 13. Kaninchen Nr. 5. Ganglienzelle aus der Hirnrinde von amöboid umgewandelten Trabantzellen umgeben (a). Im Spitzenfortsatz sind auf einer weiten Strecke Körnchen von färbbarer Substanz zu verfolgen.

Fig. 14, 15. Ganglienzellen aus der Hirnrinde, umgeben von gewucherten Trabantzellen; im Spitzenfortsatz Körnchen von färbbarer Substanz weit zu sehen.

Fig. 16. Ganglienzelle aus der Hirnrinde; der mittlere Teil des Spitzenfortsatzes enthält nur vereinzelte Körnchen der färbbaren Substanz, die im weiteren Verlauf zahlreicher auftreten.

Fig. 17. Ganglienzelle aus der Hirnrinde. Die Fortsätze sind mit Körnchen von färbbarer Substanz ausgefüllt; der Zellkörper ist zwischen 3 gewucherten Trabantzellen ( $\mathrm{T}$ ) eingeklemmt.

Fig. 18-21. Kaninchen Nr. 6. Ganglienzellen aus den Vorderhörnern des Rückenmarks. Silbermethode von Bielschowsky. Die fibrilläre Struktur ist leidlich gut erhalten, besonders in den Dendriten. Der Zellkörper enthält Vakuolen.

\section{Tafel XVII.}

Alkoholfixierung. Thioninfärbung in Celloidin eingebetteter Schnitte. Vergrößerung: Fig. 22 - Leitz, Immersion, Okul. 3. Fig. 23-25, 36-39-Zeiß, Immersion 1/12, Kompens. Okul. 6. Fig. 26-35 - Leitz, Immersion, Okular 3.

Fig. 22. Kaninchen Nr. 4. Die Körnerschicht (K) zeigt keine Veränderungen; zwischen derselben und der Purkinjeschen Zellschicht liegen einige amöboide Gliazellen (a). In der Schicht der Purkinjeschen Zellen sehen wir die Purkinjezellen in verschiedenen Stadien des Zerfalls; von einer Zelle (P) ist kaum noch ein Schatten zurückgeblieben, und die Stelle der Zelle ist durch gewucherte Gliazellen ersetzt. Auch in dieser Schicht kommen einzelne amöboide Gliazellen vor (a).

In der Molekularschicht (M) sehen wir eine normale, große, von 10 Trabantzellen umgebene Ganglienzelle (g) (um die gegenseitigen VerhäItnisse der größeren und kleineren Ganglienzellen der Molekularschicht anschaulicher zu machen, wurde diese Ganglienzelle aus einer anderen Stelle desselben Präparates und derselben Schicht hier in die Zeichnung eingeschoben); ferner sind in dieser Schicht drei normale, kleinere Gan. glienzellen (k) und 2 Gan rlienzellen $(\mathrm{x})$ zu sehen, welche eine eigenartige Degeneration aufweisen. Ähnlichen Körnchen begegnen wir ebentalls im Dendrit der nebenanliegenden Nervenzelle.

Fig. 23, 23 a, 24, 25, 36, 37, 38, 39. Gliosarcoma cerebelli beim Menschen. Ganglienzellen aus der Molekularschicht des Kleinhirns, aus der Umgebung des Tumors, die eine eigenartige Degenerationsform aufweisen; der Spitzenfortsatz ist noch erhalten, der Zellkern ist bedeutend dunkler als sonst, die Kernmembran ist in Körnchen zerfallen. Fig. 25, der gleiche 
charakteristische Kern; die Fortsätze sind verschwunden; die ganze Zelle wird einer Plasmazelle ähnlich. Fig. 39. Ein schmaler protoplasma. tischer Fortsatz ist noch sichtbar; das perinucleäre Plasma beginnt zu zerfallen, schließlich bleibt von der ganzen Zelle nur noch ein charakteristischer, maulbeerartiger Kern zurück, in welchem öfters Einkerbungen vorkommen $(23 \mathrm{a}, 24,36,37)$; vom Plasma dagegen wird nur ein schmälerer (38) oder breiterer Saum erhalten $(23 \mathrm{a}, 24,36,37)$.

Fig. 26--35. Ganglienzellen aus der Molekularschicht eines Kaninchenkleinhirns. Fig. 34-35. Normale Ganglienzellen aus der Molekularschicht des Kleinhirns eines gesunden Kaninchens. Fig. 26-33. Dieselben Ganglienzellen aus der Molekularschicht eines mit Thyreoidin vergifteten Kaninchens (Kaninchen Nr. 6), dieselbe eigenartige Degenerationsform aufweisend, welche in der Fig. $22(\mathrm{x})$ und beim Gliosarkom des Kleinhirns beim Men. schen (Taf. XVII, Fig. 23-25, 36-39) beobachtet wurde. In sämtlichen dieser Zellen ist eine typische Kernveränderung zu beobachten; in einigen sind die Fortsätze noch mehr oder weniger erhalten $(26,28,31,33)$.

Fig. 30. Das perinucleäre Plasma ist in Zerfall begriffen.

Fig. 26, 27, 28, 31. Infolge von Plasmazerfall entstand ein leerer perinucleärer Raum.

Fig. 32. Die ganze Zelle ist in Zerfall begriffen.

\section{Tafel XVIII.}

Alkoholfixierung. Thioninfärbung in Celloidin eingebetteter Schnitte. Vergrößerung: Fig. 40-49- Leitz, Immersion, 1/12. Okul. 3.

Fig. 40-46. Kaninchen Nr. 6. Akute Thyreotoxikose. 1-, 2- und 3 kernige, in verschiedenen Zerfallstadien begriffene Nervenzellen aus dem Ganglion s y m pathicum (aus dem Grenzstrang des N. sympathicus). Fig. 40, 44. Beginn der Vakuolenbildung an der Peripherie. Fig. 41-43. Beinahe das ganze Zellplasma ist vakuolisiert. Fig. 42. Kern noch erhalten, Zellkörper fast gänzlich zerfallen; im Körper sind 4 Kapselzellen zu sehen. Fig. 45, 46. Konturen der zerfallenen Zelle kaum angedeutet. Der ganze Körper ist mit gewucherten Kapselzellen ausgefüllt.

Fig. 47-49. Kaninchen Nr. 5. Akute Thyreotoxikose. Ganglienzellen aus dem Spinalganglion.

Fig. 47. Zelle von beinahe normalem Aussehen. Die Kernmembran ist nicht mehr sichtbar, der Kern hebt sich kaum vom Zellkörper ab.

Fig. 48. Der stark veränderte Zellkern fließt mit dem Körper zusammen, dessen Plasma zahlreiche Vakuolen aufweist.

Fig. 49. Zwei stark veränderte Spinalganglienzellen. Kein Kern ist mehr zu sehen; vom Körper sind nur noch Spuren erhalten. Kapselzellen sind ins Zellinnere eingedrungen und wuchern daselbst.

\section{Tafel XIX.}

Formalinfixierung. Färbung von Gefrierschnitten nach Mann-Alzheimer. Vergrößerung: Fig. 50 - Zeiß, Immersion 1/12, Okul. 3. Fig. 51. Leitz, Immersion, Okul. 3. Fig. 52. Leitz, Immersion, Okul. 5. Fig. 53-55: Reichert, Immersion, Okul. 3.

Fig. 50. Kaninchen Nr. 6. Akute Thyreotoxikose. Längsschnitt durch die weiße Rückenmarsubstanz. Die Achsenzylinder sind zum Teil erhalten, zum 
302 T. Simchowicz: Histologische Veränderungen im Nervensystem.

Teil zeigen dieselben entweder eine Anschwellung, oder körnigen Zerfall (insbesondere die feineren Achsenzylinder); sämtliche Gliazellen sind amöboid umgewandelt; in 2 amöboiden Gliazellen sind Vakuolen vorhanden.

Fig. 51. Kaninchen Nr. 3. Akute Thyreotoxikose. Amöboide Gliazelle aus der weißen Rückenmarksubstanz. Im Zellkörper ist eine Vakuole, außerdem eine Nervenfaser mit gut erhaltenem Achsenzylinder zu sehen (ax).

Fig. 52. Kaninchen Nr. 5. Akute Thyreotoxikose. Amöboide Gliazelle aus der weißen Rückenmarksubstanz mit homogenem, glänzendem, lichtbrechendem Kern.

Fig. 53, 54, 55. Kaninchen Nr. 6. Akute Thyreotoxikose. Degenerierte amöboide Gliazellen aus der weißen Rückenmarksubstanz. Im Zellplasma sehen wir zahlreiche blaue Körnchen, die entweder sehr fein (Fig. 53), oder aber bedeutend gröber (Fig. 54) und dunkler sind (Fig. 55).

\section{Tafel XX.}

Kopf eines Hundes, dem einmal $4 \mathrm{~g}$ Thyreoidin subcutan injiziert wurden. An beiden umgestülpten Ohren sind symmetrische Nekrosen zu sehen. 